\title{
miR-3133 inhibits proliferation and angiogenesis by targeting the JUNB/VEGF pathway in human umbilical vein endothelial cells
}

\author{
MENGDIE XU ${ }^{1,2^{*}}$, LIN CAO $^{1 *}$, XIAOKANG ZHANG $^{1 *}$, YU ZHUANG $^{1}$, YING ZHANG $^{1}$, \\ QINZHI WANG ${ }^{1}$, YANSU CHEN ${ }^{1}$, LICHUN XU $^{1}$ and GUIXIANG SUN ${ }^{1}$ \\ ${ }^{1}$ School of Public Health, Xuzhou Medical University, Xuzhou, Jiangsu 221004, P.R. China; \\ ${ }^{2}$ Lianyungang Center for Disease Control and Prevention, Lianyungang, Jiangsu 222003, P.R. China
}

Received December 10, 2019; Accepted April 29, 2020

DOI: $10.3892 /$ or.2020.7715

\begin{abstract}
Membranous obstruction of the inferior vena cava (MOVC) has the highest incidence rate among the different types of Budd-Chiari syndrome (BCS) in China. The inferior vena cava septum of patients with MOVC contains capillaries and the two surfaces of the membrane are composed of vascular endothelial tissue. Membrane formation occurs due to endothelial damage. MicroRNAs (miRNAs/miRs) have been verified to be involved in the pathogenesis and progression of various human diseases. A previous study by our group suggested that miR-3133 was downregulated in the serum of patients with MOVC. In the present study, the possible mechanistic implication of miR-3133 in MOVC-associated processes was further explored. It was observed that miR-3133 overexpression inhibited, whereas miR-3133 knockdown enhanced the proliferation and tube formation of human umbilical vein endothelial cells (HUVECs) using the CCK-8 and tube formation assays. JUNB, a member of activator protein 1 and an important upstream transcriptional molecule of vascular endothelial growth factor (VEGF), was proven to be a direct target gene of miR-3133 using a bioinformatics prediction and luciferase reporter assay. Meanwhile, the mRNA and protein expression of JUNB and VEGF was determined by PCR,
\end{abstract}

Correspondence to: Professor Guixiang Sun, School of Public Health, Xuzhou Medical University, 209 Tongshan Road, Xuzhou, Jiangsu 221004, P.R. China

E-mail: gxsunny@xzhmu.edu.cn

*Contributed equally

Abbreviations: MOVC, membranous obstruction of the inferior vena cava; BCS, Budd Chiari syndrome; VEGF, vascular endothelial growth factor; JUNB, JunB proto-oncogene; HUVECs, human umbilical vein endothelial cells; CCK-8, Cell Counting Kit-8

Key words: membranous obstruction of the inferior vena cava, Budd-Chiari syndrome, microRNA-3133, JUNB, vascular endothelial growth factor, proliferation, angiogenesis
ELISA and western blot analyses. Of note, miR-3133 overexpression downregulated, while miR-3133 knockdown elevated the expression of JUNB and VEGF significantly. Furthermore, it was demonstrated that JUNB upregulated the expression and secretion of VEGF to promote HUVEC proliferation and angiogenesis. miR-3133 was able to inhibit the effect of JUNB overexpression to promote cell proliferation, angiogenesis and the expression of VEGF. In conclusion, the present study demonstrated that miR-3133 regulated endothelial cell proliferation and angiogenesis through the JUNB/VEGF pathway, which may provide an approach for inhibiting diaphragm formation of the inferior vena cava in MOVC.

\section{Introduction}

Budd-Chiari syndrome (BCS), a disease named after the British physician George Budd and the Australian pathologist Hans Chiari, is a relatively rare hepatic vascular disease, which is mainly caused by obstruction of the hepatic vein outflow tract (1-3). The incidence rate of membranous obstruction of the inferior vena cava (MOVC) is the highest among all types of BCS in China; in MOVC, the diaphragm forms at the opening of the inferior vena cava and/or hepatic vein (4). This condition results in considerable physiological damage and psychological trauma and increases the economic burden of the family of affected patients.

Abnormal expressions of von Willebrand factor (vWF), endothelin-1, vascular endothelial growth factor (VEGF) and other cytokines have been detected in the plasma of patients with MOVC compared with those of normal individuals. vWF and VEGF are recognized as pro-angiogenic factors and are associated with angiogenesis $(5,6)$. Furthermore, a Chinese study reported that granulation tissue and neovascularization were present in the lesion of the inferior vena cava and that the luminal surface as covered by a layer of intact endothelial cells (7). Therefore, the formation of the inferior vena cava diaphragm possibly begins with proliferation and angiogenesis after vascular endothelial injury.

microRNAs (miRNAs/miRs) are a class of non-coding, endogenous and small RNA molecules with a length of $22-28$ nucleotides. miRNAs, as novel gene regulators, are able to 
inhibit protein production by inducing mRNA degradation or combining with the 3'-untranslated regions (UTRs) of target mRNAs to suppress the process of translation $(8,9)$. In this manner, miRNAs have a vital function in a number of pathological and physiological processes, including cell proliferation, death, immune function, apoptosis and angiogenesis $(10,11)$. A previous study by our group has indicated that the expression of miR-3133 is significantly lower in the plasma of patients with MOVC than in healthy controls (12). Therefore, downregulation of miR-3133 may be involved in the pathogenesis of MOVC through regulating the proliferation and angiogenesis of endothelial cells. However, how miR-3133 regulates the proliferation and angiogenesis of endothelial cells has remained elusive and was therefore investigated in the present study.

\section{Materials and methods}

Cell lines and reagents. Human umbilical vein endothelial cells (HUVECs; cat. no. GNhu39) were purchased from the cell bank of the Chinese Academy of Sciences. The cells were cultured in RPMI-1640 medium (KeyGen Biotech Co., Ltd.) containing $10 \%$ fetal bovine serum (Tianhang Biotechnology Co., Ltd.). The cells were incubated at $37^{\circ} \mathrm{C}$ in a humidified atmosphere with $5 \% \mathrm{CO}_{2}$.

Plasmids and transient transfections. miR-3133 mimics and their respective negative control (NC), as well as miR-3133 inhibitor and its NC, were purchased from RiboBio Biotechnology Co., Ltd. The pc-DNA3.1-3xFlag-JUNB and pc-DNA3.1-3xFlag-vector were obtained from Youbao Biotechnology Co., Ltd. The hilymax reagent was used to transiently transfect plasmids for the miR-3133 mimics and inhibitor into the cells according to the manufacturer's protocol. These transfections were performed when the HUVECs were cultured in a 12-well plate at $1.2 \times 10^{5}$ cells/well with $1 \mathrm{ml}$ fresh medium for $24 \mathrm{~h}$ and reached $60-70 \%$ confluency.

Cell proliferation assay. HUVECs were cultured in a 12-well plate at $1.2 \times 10^{5}$ cells/well with $1 \mathrm{ml}$ fresh medium for $24 \mathrm{~h}$ and transfected with plasmids after the density reached $60-70 \%$. For the proliferation assay, these transfected cells were seeded into 96 -well plate at $5 \times 10^{3}$ cells/well in $100 \mu \mathrm{l}$ fresh medium and culture was continued for $0,24,48$ and $72 \mathrm{~h}$ prior to the assay with the Cell Counting Kit-8 (CCK-8) (Dojindo).

Tube formation assay. For the tube formation assay, pre-chilled 96-well plates were coated with $50 \mu 1$ Matrigel $^{\mathrm{TM}}$ (BD Biosciences) and incubated at $37^{\circ} \mathrm{C}$ for $30 \mathrm{~min}$. Subsequently, $1 \times 10^{4}$ cells resuspended in $100 \mu \mathrm{l}$ serum-free medium were added to each well and then incubated at $37^{\circ} \mathrm{C}$ for another $6 \mathrm{~h}$ prior to capturing of images under a microscope and counting the complete tubular structures.

Reverse transcription-quantitative (RT-q)PCR assay. The total RNA was isolated from cultured cells by using TRIzol (Invitrogen; Thermo Fisher Scientific, Inc.). To quantify gene expression, the complementary DNA of miR-3133 was synthesized using the miRNA First Strand cDNA Synthesis Kit (by stem-loop; Vazyme), while the RT reaction for JUNB and
VEGF was performed using the HiScript II First Strand cDNA Synthesis Kit (Vazyme). The internal controls for miR-3133 and JUNB/VEGF were U6 and GAPDH, respectively. The 7500HT qPCR system thermal cycler (Applied Biosystems; Thermo Fisher Scientific, Inc.) was used for the amplification of the obtained complementary DNA in triplicate with AceQ qPCR SYBR-Green Master Mix (Vazyme). The program settings were as follows: Pre-denaturation at $95^{\circ} \mathrm{C}$ for $5 \mathrm{~min}$, followed by 40 cycles of $10 \mathrm{sec}$ at $95^{\circ} \mathrm{C}$, annealing at $60^{\circ} \mathrm{C}$ for $30 \mathrm{sec}$ and extension at $95^{\circ} \mathrm{C}$ for $15 \mathrm{sec}$. The relative gene expression was determined using the $2^{-\Delta \Delta \mathrm{Cq}}$ method (13). The primers used were as follows: 5'-GTCGTATCCAGT GCAGGGTCCGAGGTATTCGCACTGGATACGACATTG GG-3' (stem-loop), 5'-CGCGCGTAAAGAACTCTTAAAA-3' (forward) and 5'-AGTGCAGGGTCCGAGGTATT-3' (reverse) for miR-3133; 5'-CTCGCTTCGGCAGCACA-3' (forward) and 5'-AACGCTTCACGAATTTGCGT-3' (reverse) for U6; 5'-TGGAACAGCCCTTCTACCAC-3' (forward) and 5'-TGG AACAGCCCTTCTACCAC-3' (reverse) for JUNB; 5'-TGC CCGCTGCTGTCTAATG-3' (forward) and 5'-GCGAGTCTG TGTTTTTGCAG-3' (reverse) for VEGF; 5'-GCCGGTGCT GAGTATGTC-3' (forward) and 5'-CTTCTGGGTGGCAGT GAT-3' (reverse) for GAPDH.

Western blot analysis. Following transfection, the HUVECs were collected and lysed using radioimmunoprecipitation assay lysis buffer containing $100 \mathrm{mM}$ phenylmethylsulfonyl fluoride and ultrasound. The supernatant was collected after centrifuging at $12435 \mathrm{x} \mathrm{g}$ for $5 \mathrm{~min}$ at $4^{\circ} \mathrm{C}$ and the protein concentration was measured using a bicinchoninic acid protein assay kit (Vazyme) according to the manufacturer's protocol. Equal amounts of protein were separated by $10 \%$ SDS-PAGE and then transferred to a polyvinylidene difluoride membrane. The membranes were blocked with Tris-buffered saline containing $0.1 \%$ Tween-20 (TBST) and 5\% skimmed milk at room temperature for $2 \mathrm{~h}$. The primary antibodies, including anti-JUNB (cat. no. ab128878; 1:1,000 dilution; Abcam) and anti-VEGF (cat. no. ER30607; 1:1,000 dilution; Huabio) were incubated at $4^{\circ} \mathrm{C}$ overnight. The internal control of proteins was $\alpha$-tubulin (cat. no. 10094-1-AP; 1:1,000 dilution; Beyotime Institute of Biotechnology, Inc.). The membranes were washed three times with TBST and incubated with horseradish peroxidase (HRP)-labeled goat anti-rabbit IgG secondary antibodies (cat. no. A0208; 1:1,000 dilution; Beyotime Institute of Biotechnology, Inc.) at room temperature for $1 \mathrm{~h}$. The bands were visualized using Immobilon Western Chemiluminescent HRP Substrate (EMD Millipore). The results were analyzed by Image Lab 5.2.1 (Bio-Rad Laboratories, Inc.) and each blot was performed at least three times.

ELISA. HUVECS $\left(2.5 \times 10^{5}\right.$ cells/well $)$ were seeded into a six-well plate and the medium was replaced with $1 \mathrm{ml}$ serum-free fresh medium at $24 \mathrm{~h}$ after transfection. After another $24 \mathrm{~h}$, the supernatant was collected and centrifuged at $1,000 \mathrm{x} \mathrm{g}$ for $20 \mathrm{~min}$ at $4^{\circ} \mathrm{C}$ to perform ELISA. The human VEGF ELISA kit (Elabscience) was used to detect the concentration of VEGF according to the manufacturer's protocol.

Bioinformatics prediction and luciferase reporter assay. MicroRNA.org (www.microrna.org/microrna/; August 2010 
A
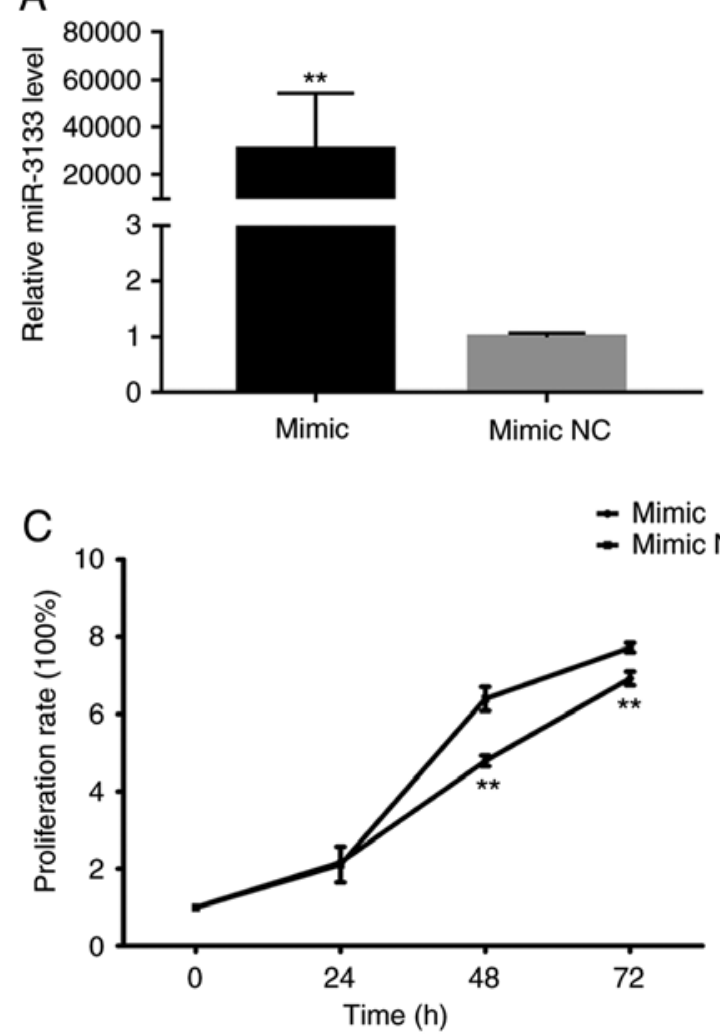

E

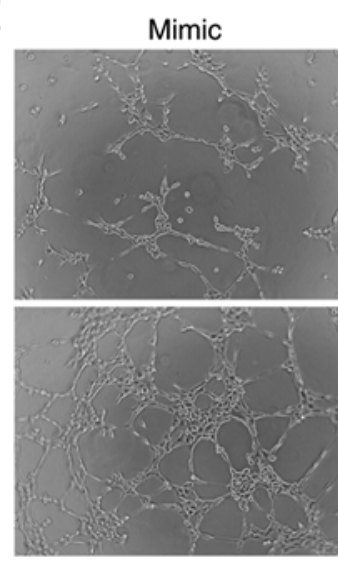

Inhibitor
Mimic NC

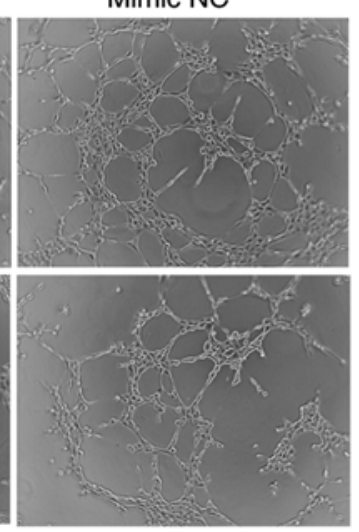

Inhibitor NC
B
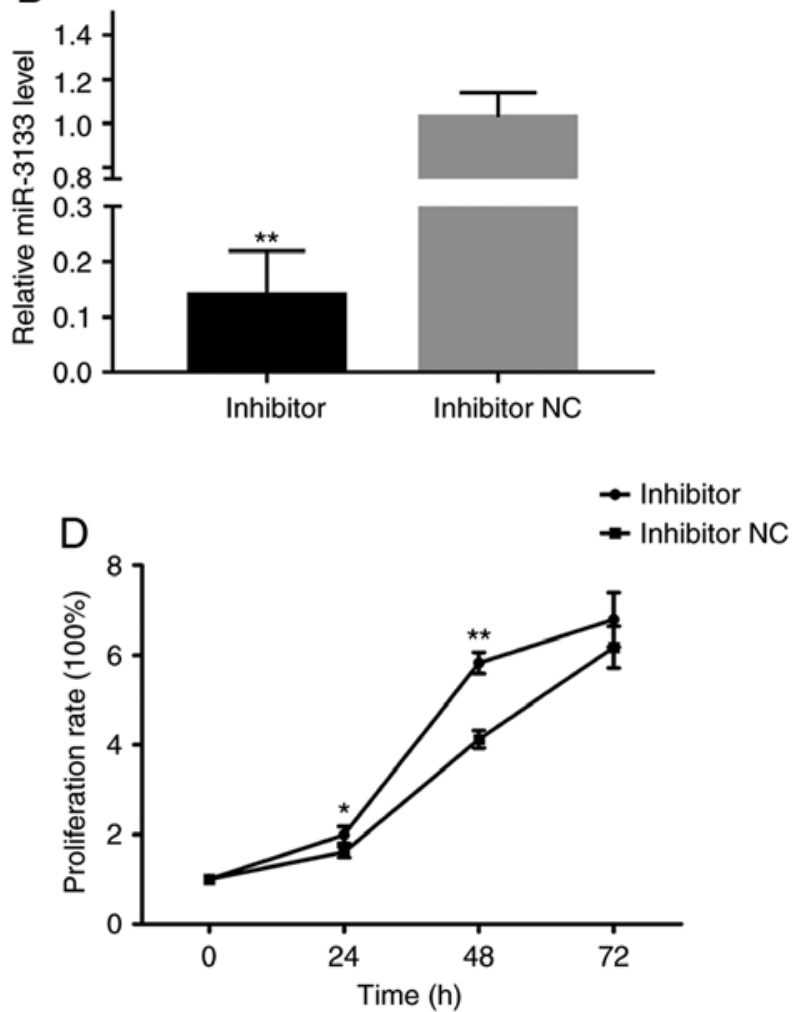

$\mathrm{F}$

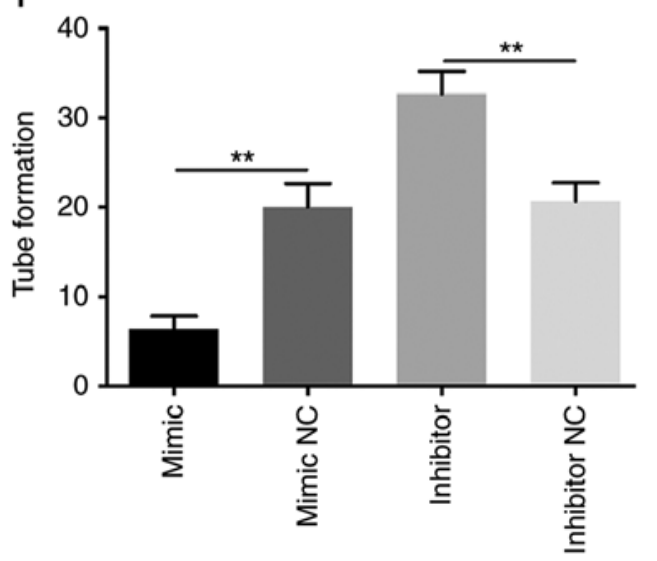

Figure 1. miR-3133 mimics inhibited, whereas miR-3133 inhibitor promoted the proliferation and angiogenesis of HUVECs. (A) RT-qPCR analysis of miR-3133 expression in HUVECs after transfection of miR-3133 mimics or NC. (B) RT-qPCR analysis of miR-3133 expression in HUVECs after transfection of miR-3133 inhibitor or NC. (C) CCK-8 assay of HUVECs transfected with miR-3133 mimics or NC. (D) CCK-8 assay of HUVECs transfected with miR-3133 inhibitor or NC. (E) Tube formation of HUVECs transfected with miR-3133 mimics, inhibitor or NC (magnification, x200). (F) The number of tubes formed ( $\mathrm{n}=3$ /group). Values are expressed as the mean \pm standard deviation. ${ }^{*} \mathrm{P}<0.05 ;{ }^{* *} \mathrm{P}<0.01$ vs. the respective control. miR, microRNA; NC, negative control; HUVECs, human umbilical vein endothelial cells; CCK-8, Cell Counting Kit-8; RT-qPCR, reverse transcription-quantitative PCR.

release; last update, 1 November 2010; Species, human) was used to predict the putative targets of miR-3133 (14). The wild-type (WT) 3'-UTR of JUNB containing predicted miR-3133 binding sequences and the mutant (Mut) 3'-UTR of JUNB were chemically produced by ABM Biotechnology Co., Ltd. The WT and Mut 3'UTR of JUNB were subcloned into the psiCHECK-2 vector. HUVECs at $5 \times 10^{4}$ cells/well in 24-well plates were co-transfected with $0.5 \mu \mathrm{g}$ reporter plasmid and miR-3133 mimics or miR-3133 mimics NC using hilymax reagent for $36 \mathrm{~h}$, and cell lysates (Promega Corp.) were then prepared according to the manfacturer's instructions. Luciferase activity was measured with a dual-Luciferase
Reporter Assay System (Promega Corp.) and the activity was normalized to the Renilla luciferase gene.

Statistical analysis. Values are expressed as the mean \pm standard deviation and analyzed with SPSS 20.0 (IBM Corp.). The two-tailed Student's t-test (two groups) or one-way analysis of variance were used to evaluate the inter-group differences. When comparing between any two means, the data satisfying the normality and the homogeneity of the variance were compared with the least significant differences t-test, and otherwise, Dunnett's T3 test was used. $\mathrm{P}<0.05$ was considered to indicate a statistically significant difference. 
A
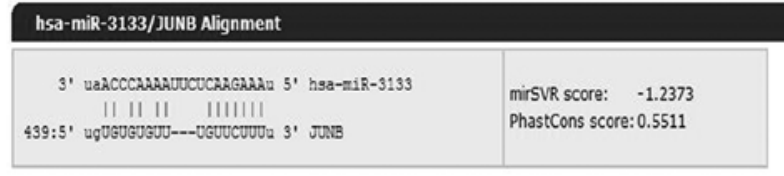

\section{hsa-miR-3133/JUNB Alignment}

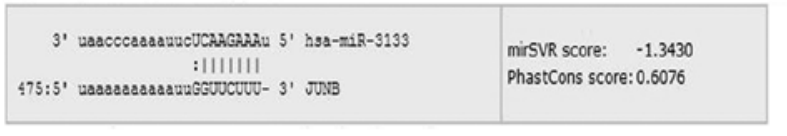

\section{C}

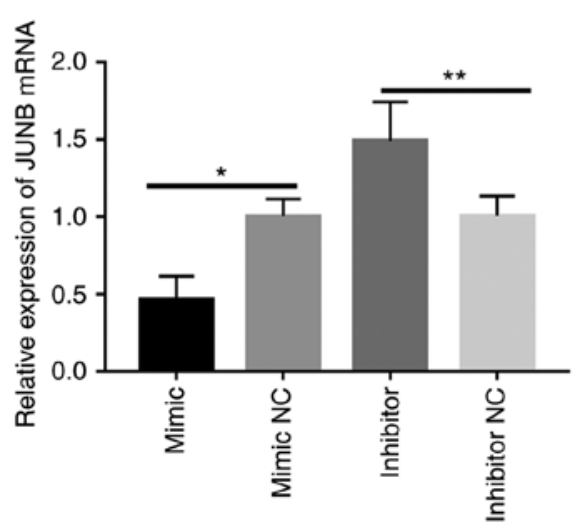

E

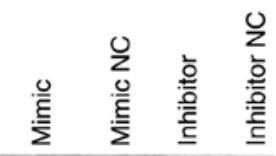

JUNB

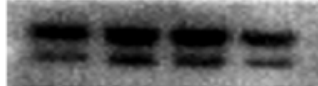

VEGF

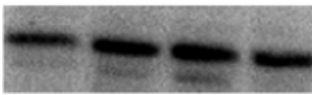

Tubulin

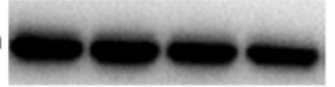

B

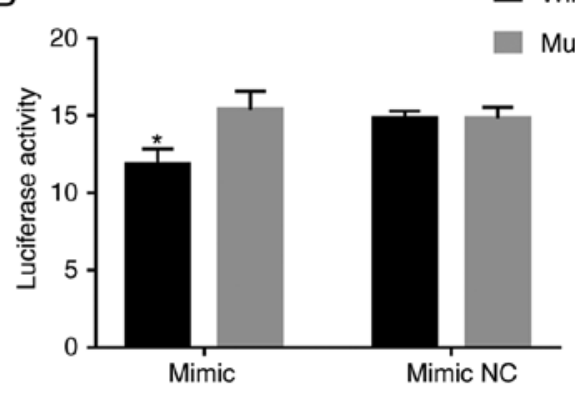

$\mathrm{D} \leftrightarrows$

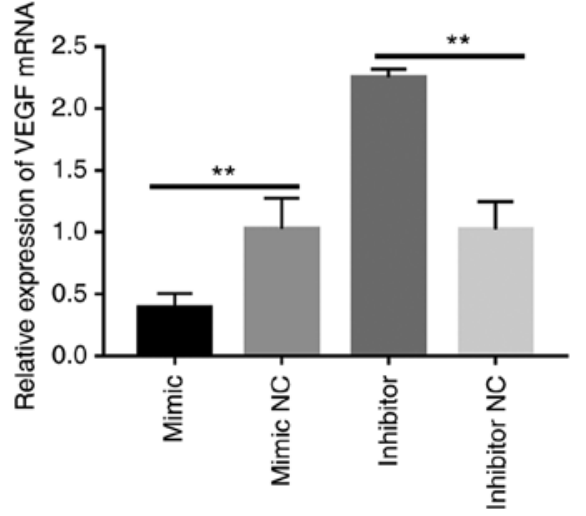

F

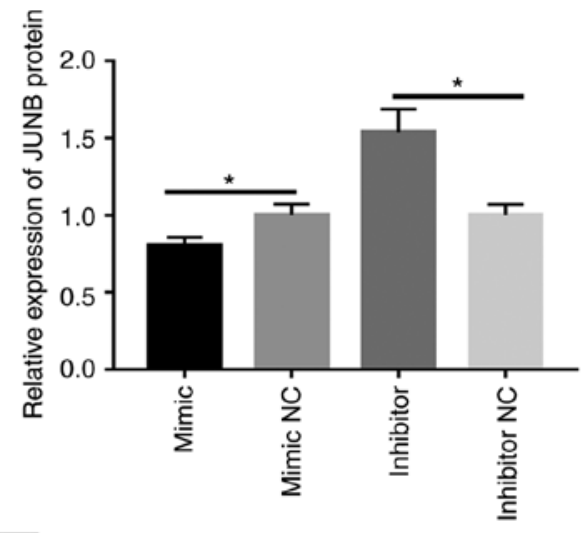

G

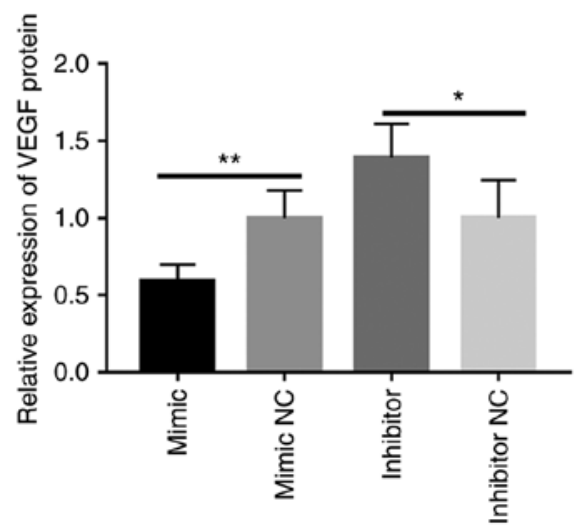

Figure 2. JUNB is a direct target of miR-3133, whereas miR-3133 inhibits the expression of JUNB and VEGF. (A) Putative hsa-miR-3133 binding and mutated sites in the 3'UTR of JUNB. (B) JUNB 3'UTR reporter activity in the presence of miR-3133 mimics or mimics NC was measured by a luciferase assay and normalized to the activity of Renilla luciferase. (C) RT-qPCR analysis of JUNB expression in HUVECs after transfection. (D) RT-qPCR analysis of VEGF expression in HUVECs after transfection. (E and F) Western blot images and analysis of JUNB and VEGF, respectively. (G) ELISA of VEGF concentration in the supernatant. Values are expressed as the mean \pm standard deviation. ${ }^{*} \mathrm{P}<0.05$ and ${ }^{* *} \mathrm{P}<0.01$ vs. the respective NC. miR, microRNA; NC, negative control; HUVECs, human umbilical vein endothelial cells; RT-qPCR, reverse transcription-quantitative PCR; VEGF, vascular endothelial growth factor; hsa, Homo sapiens; wild, wild-type; mut, mutant; UTR, untranslated region.

\section{Results}

miR-3133 regulates the proliferation and angiogenesis of HUVECs. miR-3133 mimics, inhibitor and their respective
NCs were transfected into cells using the hilymax reagent. RT-qPCR demonstrated that the mimics increased the levels of miR-3133, whereas the inhibitor reduced the levels of miR-3133 (Fig. 1A and B). The results of the CCK-8 assay 

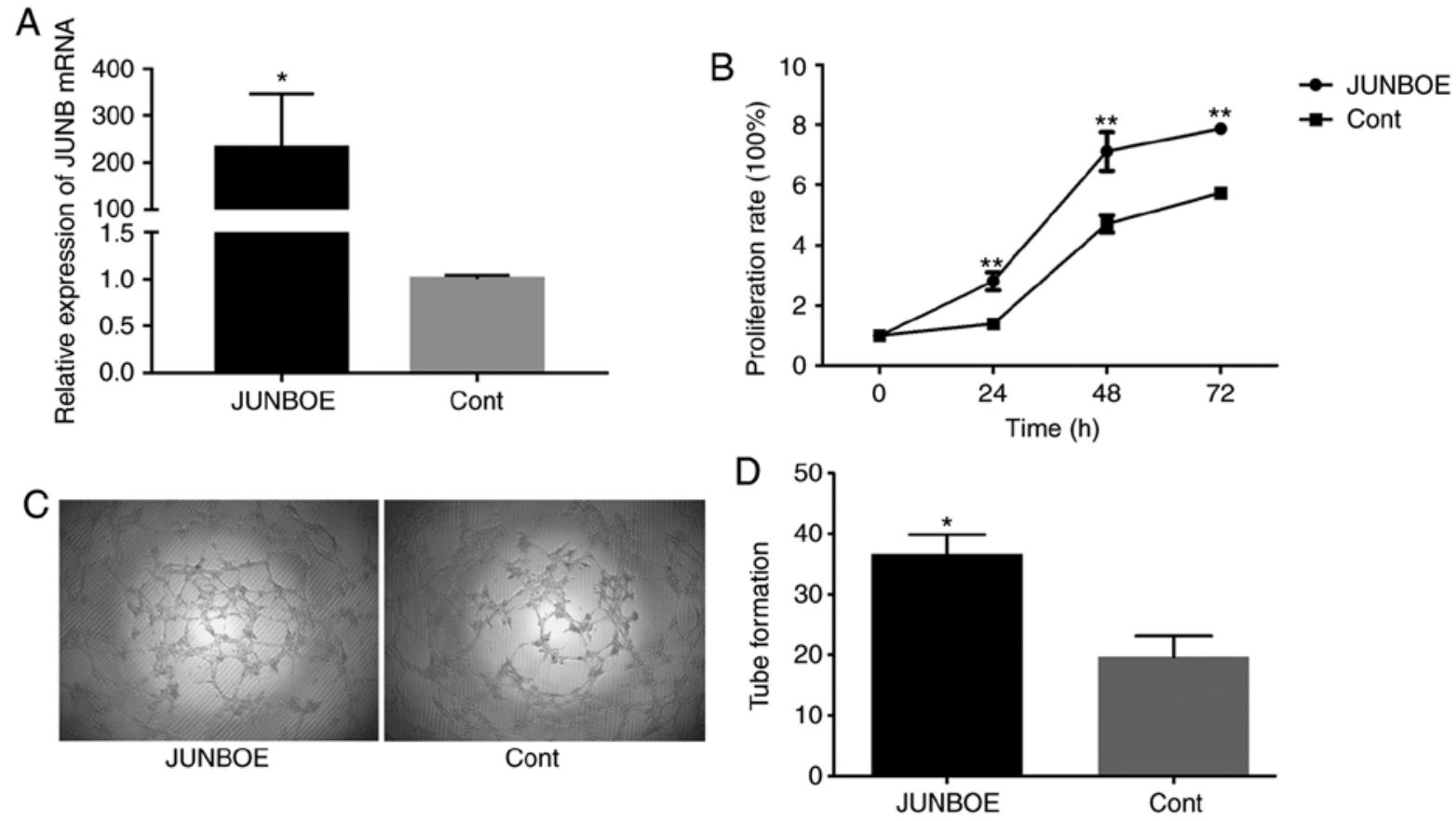

Figure 3. JUNB overexpression promotes endothelial cell proliferation and angiogenesis. (A) Reverse transcription-quantitative PCR analysis of JUNB expression in HUVECs after transfection of two plasmids (JUNBOE or Cont). (B) Cell Counting Kit-8 assay of HUVECs transfected with JUNBOE or Cont. (C) Images of the tube formation of HUVECs transfected with JUNBOE or Cont (magnification, $\mathrm{x} 200$ ). (D) The number of tubes formed ( $\mathrm{n}=3 / \mathrm{group}$ ). Values are expressed as the mean \pm standard deviation. ${ }^{*} \mathrm{P}<0.05 ;{ }^{* *} \mathrm{P}<0.01$ vs. the respective control. Cont, control; HUVECs, human umbilical vein endothelial cells; JUNBOE, JUNB overexpression.

indicated that, compared with their respective $\mathrm{NCs}$, the miR-3133 mimics attenuated the proliferation of HUVECs, whereas the miR-3133 inhibitor promoted it (Fig. 1C and D). In addition, the tube formation assay demonstrated that the miR-3133 mimics reduced the formation of tube-like structures, whereas miR-3133 inhibitor caused their stimulation (Fig. 1E and F). Taken together, these results indicated that miR-3133 suppresses proliferation and angiogenesis.

JUNB is a target gene of $m i R-3133$. MicroRNA.org was used to predict target genes of miR-3133 to study the molecular mechanism of miR-3133. The mirSVR score and PhastCons score are the indicators in the miRanda evaluation. Genes with a lower mirSVR score and a higher PhastCons score were more likely to be a target gene of miR-3133. The prediction result suggested that JUNB (mirSVR score $=-1.2373$, PhastCons score $=0.5511$ ) was a potential target gene of miR-3133 (Fig. 2A). Subsequently, a dual-luciferase assay was performed, revealing that the luciferase activity in cells cotransformed with miR-3133 mimics and wild-type JUNB was significantly reduced compared with that in cells cotransformed with miR-3133 mimics and mutated JUNB. However, the luciferase activity in cells cotransformed with miR-3133 mimics NC and wild-type JUNB was not significantly different compared with that in cells cotransformed with miR-3133 mimics NC and mutant-type JUNB (Fig. 2B).

miR-3133 inhibits the expression of JUNB and VEGF in $H U V E C s$. After transfection, the expression of JUNB and VEGF was detected at the mRNA and protein level by RT-qPCR and western blot analysis, respectively. The results indicated that, compared with the respective $\mathrm{NC}$, the expression levels of JUNB and VEGF were significantly decreased in the mimics group and were higher in the inhibitor group (Fig. 2C-F). In addition, ELISA was used to detect the secretion levels of VEGF after transfection, and the results suggested that, compared with the respective control group, the secretion of VEGF was reduced in the miR-3133 mimics group and upregulated in the miR-3133 inhibitor group (Fig. 2G). These results demonstrate that miR-3133 is able to negatively regulate the expression of JUNB and VEGF.

Overexpression of JUNB promotes proliferation and angiogenesis in HUVECs. The forced expression of JUNB in endothelial cells was previously reported to stimulate the formation of a tip-like cell morphology and angiogenesis and induces VEGF expression (15-17). In the present study, it was assessed whether JUNB has a role in the proliferation and angiogenesis of HUVECs. The JUNB overexpression plasmid (JUNBOE) was constructed and the empty vector (Cont) was used as a control. The expression of JUNB was detected using RT-qPCR to determine whether the overexpression with JUNBOE was effective. The results indicated that, compared with the Cont group, JUNB was highly expressed in the JUNBOE group (Fig. 3A). The CCK-8 assay was then used to detect cell proliferation after transfection and tube formation was evaluated by a tube formation assay. The results suggested that the proliferation of HUVECs in the JUNBOE group was higher than that in the Cont group at $24 \mathrm{~h}$ after transfection (Fig. 3B). In addition, the tube formation ability in the JUNBOE group increased compared with that in the Cont group (Fig. 3C and D).

$J U N B$ positively regulates the expression and secretion of $V E G F$ in HUVECs. The expression of VEGF at the mRNA 

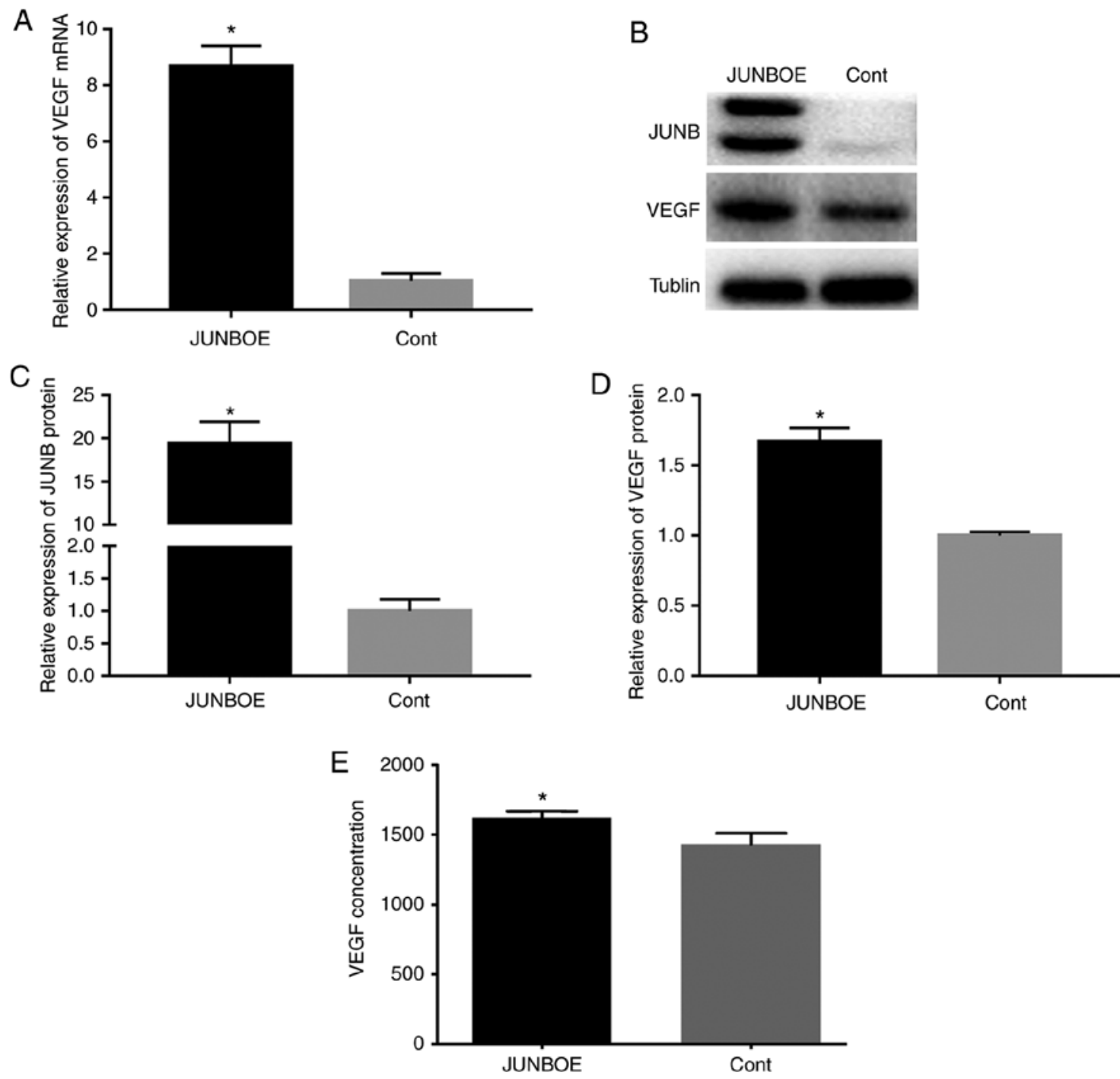

Figure 4. JUNB positively regulates the expression and secretion of VEGF in HUVECs. (A) Reverse transcription-quantitative PCR analysis of VEGF expression in HUVECs after transfection of two plasmids (JUNBOE or Cont). (B) Western blot image and quantitative analysis of (C) JUNB and (D) VEGF after transfection with JUNBOE or Cont, respectively. (E) ELISA of VEGF concentration in the supernatant after transfection with JUNBOE or Cont. Values are expressed as the mean \pm standard deviation. ${ }^{*} \mathrm{P}<0.05$ vs. the respective control. Cont, control; HUVECs, human umbilical vein endothelial cells; JUNBOE, JUNB overexpression; VEGF, vascular endothelial growth factor.

and protein levels was assessed after transfection with JUNBOE or Cont to verify the role of JUNB in the regulation of VEGF. The results indicated that the mRNA (Fig. 4A) and protein expression levels (Fig. 4B-D) of VEGF increased when JUNB was overexpressed. Furthermore, JUNB overexpression induced the secretion of VEGF (Fig. 4E).

miR-3133 reduces the proliferation and angiogenesis ability of HUVECs promoted by JUNB overexpression. miR-3133 mimics or miR-3133 mimics NC was transfected into cells together with JUNBOE or Cont. A total of four groups were included in the experiment, namely mimics $\mathrm{NC}+\mathrm{Cont}$, mimics+Cont, mimics NC+JUNBOE and mimics+JUNBOE. The results of the CCK-8 assay indicated that the mimics NC+JUNBOE group had the highest cell proliferation rate, whereas the mimics+Cont group had the lowest rate (Fig. 5A). In addition, in the tube formation assay, the number of tubular structures in the four groups exhibited the same trend (Fig. 5B and C). This result indicated that miR-3133 partially inhibited the positive regulation of proliferation and angiogenesis by JUNB.

miR-3133 abrogates the induction of VEGF expression caused by overexpression of JUNB. VEGFA expression was detected at the mRNA and protein level by RT-qPCR and western blot analysis, respectively. The results indicated that compared with the mimics NC+control group, the expression of VEGFA was markedly upregulated in the mimics $\mathrm{NC}+\mathrm{JUNBOE}$ group, while it was decreased in the mimics+Cont group. Co-transfection with mimics abrogated the effect of JUNBOE to upregulate VEGFA. Furthermore, JUNB was downregulated in the mimics+JUNBOE group compared with the mimics NC+JUNBOE group, which indicates that miR-3133 exerts its inhibitory effect on VEGFA expression via inhibiting the expression of JUNB (Fig. 6A-C). In addition, the results of the ELISA revealed that, compared with the mimics+Cont group, the concentration of VEGFA secreted into the culture medium by HUVECs after cotransformation of miR-3133 


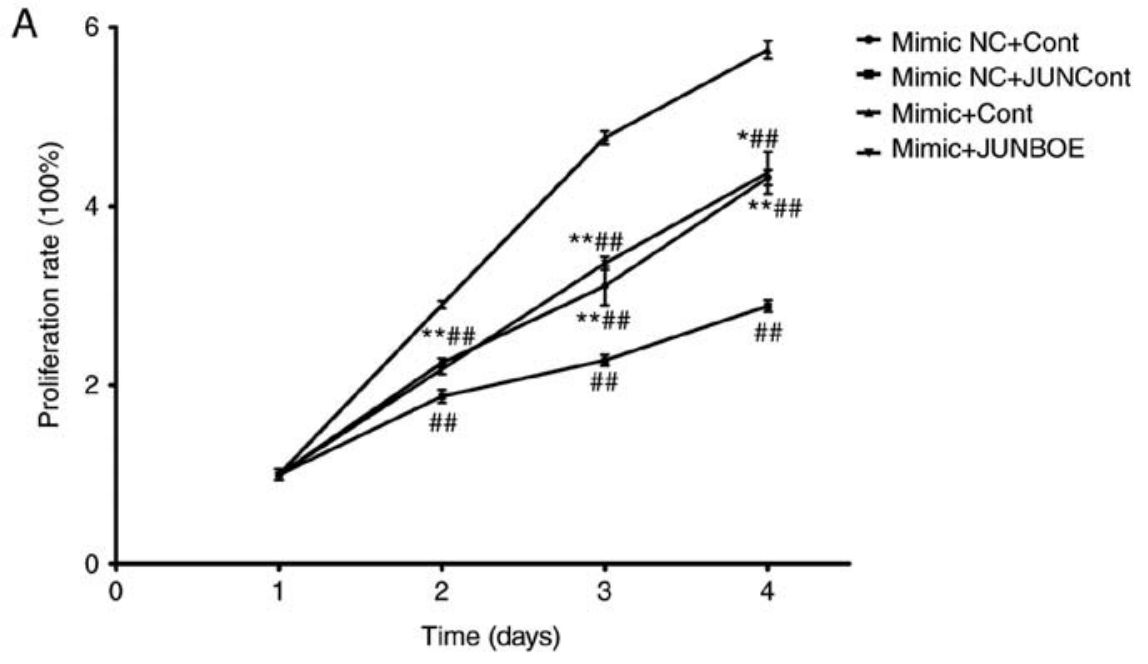

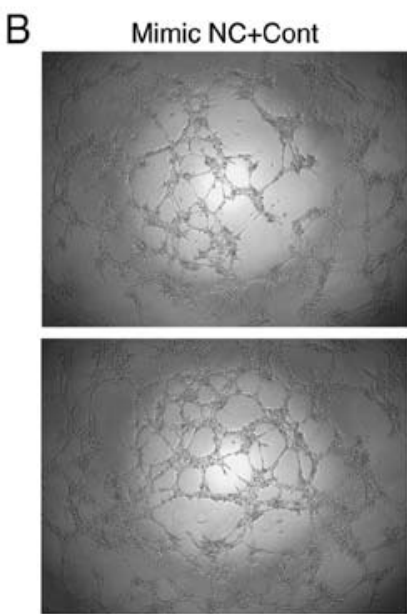

Mimic NC+JUNBOE

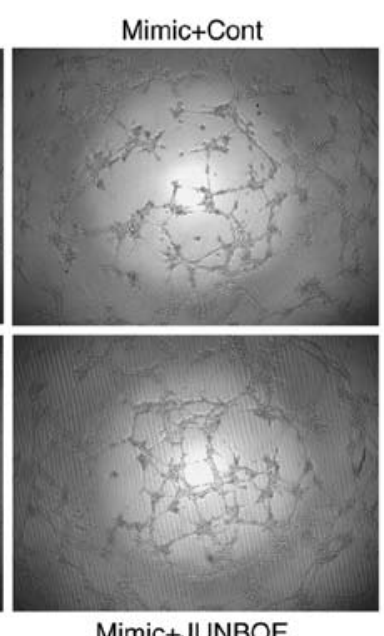

Mimic+JUNBOE

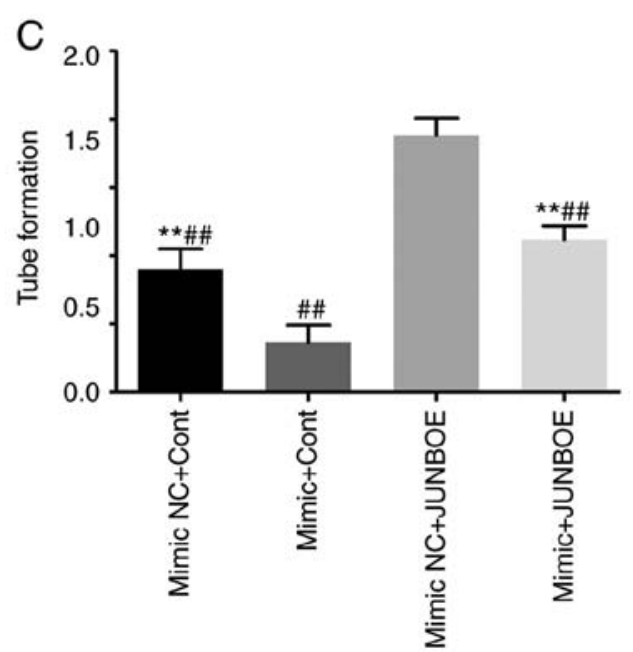

Figure 5. miR-3133 inhibits the positive regulation of proliferation and angiogenesis by JUNB to a certain extent. (A) Cell Counting Kit-8 assay of HUVECs after cotransformation of miR-3133 and JUNBOE. (B) Images of the tube formation of HUVECs after cotransformation of miR-3133 and JUNBOE (magnification, $\mathrm{x} 200$ ). (C) The number of tubes formed ( $\mathrm{n}=3$ /group). Values are expressed as the mean \pm standard deviations. ${ }^{*} \mathrm{P}<0.05$; ${ }^{* * *} \mathrm{P}<0.01$ vs. mimics+Cont; ${ }^{\#} \mathrm{P}<0.01$ vs. mimics NC+JUNBOE. Cont, control; NC, negative control; HUVECs, human umbilical vein endothelial cells; JUNBOE, JUNB overexpression; miR, microRNA.

and/or JUNBOE was markedly upregulated in the mimics NC+JUNBOE group (Fig. 6D).

\section{Discussion}

BCS is a blood reflux disorder caused by complete or incomplete obstruction of the hepatic vein outflow tract or inferior vena cava hepatic segment. Its clinical manifestations are mainly portal hypertension or inferior vena cava hypertension $(18,19)$. From a global perspective, BCS is a rare disease, but a large number of cases are reported in relatively poor countries, including China, India, South Africa and Nepal, whereas relatively few cases are reported in Western countries (20). BCS is a complex process involving numerous factors and the pathogenesis and epidemiology exhibit marked differences between China and Western countries (21). In China, MOVC is the most common type of BCS. However, the specific pathogenesis remains to be fully elucidated. Therefore, the present study aimed to preliminarily assess the possible molecular mechanisms of the occurrence of MOVC.
Riemens et al (22) reported that the inferior vena cava septum is composed of small pieces of fibrous tissue, which contain capillaries, and the surface is covered with endothelial cells. The two surfaces of the membrane are composed of vascular endothelial tissues and membrane formation occurs due to endothelial damage $(4,23)$. Therefore, abnormal proliferation and angiogenesis of HUVECs are the key factors involved in membranous formation.

Angiogenesis refers to the formation of new blood vessels by the development of existing capillaries or post-capillary veins; it includes the following processes: Degradation of the vascular basement membrane; activation, proliferation and migration of vascular endothelial cells; and reconstruction of new blood vessels and vascular network (24).

Angiogenesis is a complex process that relies on the coordination of angiogenic and inhibitory factors. Under normal circumstances, these factors are in equilibrium. Once this balance is broken, the vascular system is activated, causing excessive angiogenesis or inhibiting the vascular system to degenerate blood vessels. VEGF is a heparin-binding angiogenic growth factor displaying high specificity for vascular 

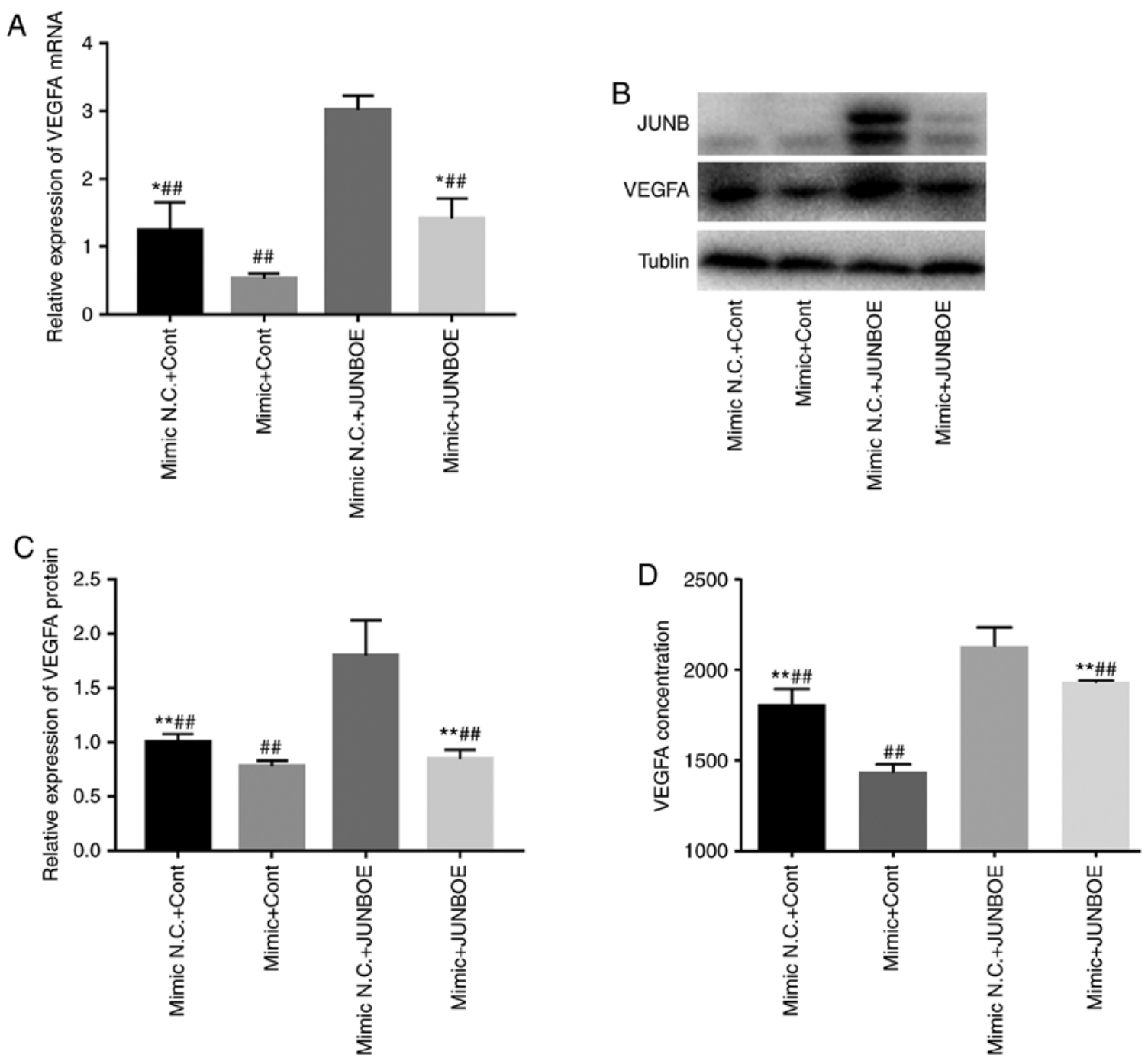

Figure 6. miR-3133 partially inhibits the effect of JUNB on the expression and secretion of VEGF. (A) Reverse transcription-quantitative PCR analysis of VEGF expression in human umbilical vein endothelial cells after cotransformation of miR-3133 and JUNBOE. (B and C) Western blot images and analysis of VEGF after cotransformation of miR-3133 and JUNBOE. (D) ELISA of VEGF concentration in the supernatant after cotransformation of miR-3133 and JUNBOE. Values are expressed as the mean \pm standard deviation. ${ }^{*} \mathrm{P}<0.05 ;{ }^{* * *} \mathrm{P}<0.01$ vs. mimics+Cont; ${ }^{\# \#} \mathrm{P}<0.01$ vs. mimics NC+JUNBOE. Cont, control; NC, negative control; HUVECs, human umbilical vein endothelial cells; JUNBOE, JUNB overexpression; miR, microRNA.

endothelial cells and regulates proliferation, migration and angiogenesis (25-27). As a key regulator to sustain endothelial function, VEGF correlates with the thrombus organization and modulates the function of vascular endothelial cells $(25,26,28)$. In a preliminary study by our group, the plasma samples from nine patients with MOVC and five healthy control were analyzed (12). Analysis of these samples indicated that the serum concentration of VEGF in patients with MOVC was increased compared with that in subjects without BCS or any other types of BCS.

In the human genome, almost one-third of the genes are thought to be regulated by miRNAs. miRNAs function as important endogenous regulators of gene expression and are thereby implicated in modulating various biological processes through complementary miRNA-mRNA binding to form silencing complexes (25,29-35). Recent evidence has indicated that the dysregulation of miRNA is involved in numerous pathological processes (36-38), and miRNA inhibitors have been demonstrated to have tremendous therapeutic potential in a multitude of studies $(39,40)$. Therefore, continuous exploration of the roles of miRNAs will provide possible therapeutic targets for MOVC.

A previous study by our group reported that a series of miRNAs was abnormally expressed in patients with MOVC (12). Among these miRNAs, miR-3133 was significantly downregulated. Thus, in the present study, miR-3133 mimics, mimics NC, inhibitor and inhibitor NC were individually transfected into HUVECs to detect the association between miR-3133 and processes of septum formation. A subsequent CCK-8 assay demonstrated that miR-3133 mimics attenuated the proliferation HUVECS, whereas miR-3133 inhibitor promoted the proliferation as compared with the respective NC. In addition, the tube formation assay demonstrated that miR-3133 mimics reduced, whereas miR-3133 inhibitor stimulated the formation of tube-like structures by HUVECs. Taken together, miR-3133 has an inhibitory role in the proliferation and angiogenesis of HUVECs.

The results of MicroRNA.org and a dual-Luciferase reporter assay proved that JUNB was a target gene of miR-3133. Furthermore, miR-3133 was able to negatively regulate JUNB and VEGF at the mRNA and protein level. In addition, ELISA 
demonstrated that the secretion of VEGF by HUVECs was reduced following transfection with miR-3133 overexpression vector. Yoshitomi et al (15) and Ryzhov et al (41) proved that JUNB not only positively regulates VEGF at the mRNA and protein level but also promotes VEGF secretion. The proliferative and angiogenic capacity of cells was enhanced when JUNB was overexpressed. The inhibitory effects of miR-3133 mimics on the proliferation and angiogenic capacity were abrogated by co-transfection with JUNB, which indicated that miR-3133 inhibited the expression of JUNB so that it could not exert its role in promoting proliferation and angiogenesis and reduced the expression and secretion of VEGF.

In conclusion, the present study indicated that miR-3133 regulated the angiogenesis potential of HUVECs through the JUNB/VEGF pathway, and may have an important role in membrane formation as a key pathogenic process of MOVC. However, this is only a possible mechanism. In order to further validate the angiogenic role of miR-3133 in MOVC in future studies, it will be attempted to examine pathological HUVECs, plasma samples and their corresponding clinical features to study the expression levels of miR-3133 in pathological HUVECs and the association between miR-3133 and the disease severity.

\section{Acknowledgements}

Not applicable.

\section{Funding}

The present study was supported by a grant from the National Natural Science Foundation of China (grant no. 81872647).

\section{Availability of data and materials}

All data generated or analyzed during this study are included in this published article.

\section{Authors' contributions}

GS, LX and YC conceived and designed the experiments. MX, LC, XZ, YuZ, YiZ and QW performed the experiments. MX, LC and XZ analyzed the experimental data and wrote the manuscript. All authors read and approved the final manuscript.

\section{Ethics approval and consent to participate}

Not applicable.

\section{Patient consent for publication}

Not applicable.

\section{Competing interests}

The authors declare that they have no competing interests.

\section{References}

1. Menon KV, Shah V and Kamath PS: The Budd-Chiari syndrome. N Engl J Med 350: 578-585, 2004.
2. Martens P and Nevens F: Budd-Chiari syndrome. United European Gastroenterol J 3: 489-500, 2015.

3. Zanetto A,Pellone M and Senzolo M: Milestones in the discovery of Budd-Chiari syndrome. Liver Int 39: 1180-1185, 2019.

4. Dang X, Li L and Xu P: Research status of Budd-Chiari syndrome in China. Int J Clin Exp Med 7: 4646-4652, 2014.

5. Yuan W, Qian M, Li ZX, Zhao CL, Zhao J and Xiao JR: Endothelin-1 activates the notch signaling pathway and promotes tumorigenesis in giant cell tumor of the spine. Spine (Phila Pa 1976) 44: E1000-E1009, 2019.

6. Tsao CJ, Pandolfi L, Wang X, Minardi S, Lupo C, Evangelopoulos M, Hendrickson T, Shi A, Storci G, Taraballi F and Tasciotti E: Electrospun patch functionalized with nanoparticles allows for spatiotemporal release of VEGF and PDGF-BB promoting in vivo neovascularization. ACS Appl Mater Interfaces 10: 44344-44353, 2018.

7. Qiao SS, Dang XW, Xu DQ, Wu Y, Li J, Zhang HX, Chen KS, Xu PQ and Zhang SJ: Morphological features of pathological membrane of inferior vena cava associated with Budd-Chiari syndrome. Chin J Exp Sur 29: 1598-1600, 2012.

8. He L and Hannon GJ: MicroRNAs: Small RNAs with a big role in gene regulation. Nat Rev Genet 5: 522-531, 2004.

9. Kontomanolis EN and Koukourakis MI: MicroRNA: The potential regulator of endometrial carcinogenesis. Microrna 4: 18-25, 2015.

10. Urbich C, Kuehbacher A and Dimmeler S: Role of microRNAs in vascular diseases, inflammation, and angiogenesis. Cardiovasc Res 79: 581-588, 2008.

11. Hwang HW and Mendell JT: MicroRNAs in cell proliferation, cell death, and tumorigenesis. Br J Cancer 96 (Suppl): R40-R44, 2007.

12. Sun GX, Su Y, Li Y, Zhang YF, Xu LC, Zu MH, Huang SP, Zhang JP and Lu ZJ: Circulating microRNA profile in patients with membranous obstruction of the inferior vena cava. Exp Ther Med 11: 811-817, 2016.

13. Livak KJ and Schmittgen TD: Analysis of relative gene expression data using real-time quantitative PCR and the 2(-Delta Delta $\mathrm{C}(\mathrm{T})$ ) method. Methods 25: 402-408, 2001.

14. Enright AJ, John B, Gaul U, Tuschl T, Sander C and Marks DS: MicroRNA targets in Drosophila. Genome Biol 5: R1, 2003.

15. Yoshitomi Y, Ikeda T, Saito H, Yoshitake Y, Ishigaki Y, Hatta T, Kato $\mathrm{N}$ and Yonekura $\mathrm{H}$ : JunB regulates angiogenesis and neurovascular parallel alignment in mouse embryonic skin. J Cell Sci 130: 916-926, 2017.

16. Zou Y, Li Q, Xu Y, Yu X, Zuo Q, Huang S, Chu Y, Jiang Z and Sun L: Promotion of trophoblast invasion by lncRNA MVIH through inducing Jun-B. J Cell Mol Med 22: 1214-1223, 2018.

17. Sadri D, Farhadi S and Nourmohamadi P: Angiogenesis in odontogenic keratocyst and dentigerous cyst: Evaluation of JunB and VEGF expression. Dent Res J (Isfahan) 16: 327-332, 2019.

18. Dang XW, Xu PQ, Ma XX, Xu DQ, Zhu YJ and Zhang YS: Surgical treatment of Budd-Chiari syndrome: Analysis of 221 cases. Hepatobiliary Pancreat Dis Int 10: 435-438, 2011.

19. $\mathrm{Li} \mathrm{SL}, \mathrm{Zu} \mathrm{MH}$ and $\mathrm{Lu} \mathrm{ZJ}$ : A review on the research status and trends of Budd-Chiari syndrome. Zhonghua Liu Xing Bing Xue Za Zhi 31: 1192-1195, 2010 (In Chinese).

20. Wang ZG, Zhang FJ, Yi MQ and Qiang LX: Evolution of management for Budd-Chiari syndrome: A team's view from 2564 patients. ANZ J Surg 75: 55-63, 2005.

21. Qi XS, Guo XZ and Fan DM: Difference in Budd-Chiari syndrome between the West and China. Hepatology 62: 656, 2015.

22. Riemens SC, Haagsma EB, Kok T, Gouw AS and van der Jagt EJ: Familial occurrence of membranous obstruction of the inferior vena cava: Arguments in favor of a congenital etiology. J Hepatol 22: 404-409, 1995

23. Teng F, Zu MH and Hua QJ: Correlations of iodide ions with vascular endothelial growth factor and its receptors during the proliferation of vascular endothelial cells. Genet Mol Res 13: 6439-6447, 2014

24. Carmeliet P and Jain RK: Molecular mechanisms and clinical applications of angiogenesis. Nature 473: 298-307, 2011.

25. Chamorro-Jorganes A, Lee MY, Araldi E, Landskroner-Eiger S, Fernández-Fuertes M, Sahraei M, Quiles Del Rey M, van Solingen C, Yu J,Fernández-Hernando C, et al: VEGF-induced expression of miR-17-92 cluster in endothelial cells is mediated by ERK/ELK1 activation and regulates angiogenesis. Circ Res 118: 38-47, 2016

26. Ferrara N, Gerber HP and LeCouter J: The biology of VEGF and its receptors. Nat Med 9: 669-676, 2003. 
27. Shibuya M: Vascular endothelial growth factor and its receptor system: Physiological functions in angiogenesis and pathological roles in various diseases. J Biochem 153: 13-19, 2013.

28. Kim S, Jun JH, Kim J, Kim DW, Jang YH, Lee WJ, Chung HY and Lee SJ: HIF-1 $\alpha$ and VEGF expression correlates with thrombus remodeling in cases of intravascular papillary endothelial hyperplasia. Int J Clin Exp Pathol 6: 2912-2918, 2013.

29. Zhou XL, Wu JH, Wang XJ and Guo FJ: Integrated microRNA-mRNA analysis revealing the potential roles of microRNAs in tongue squamous cell cancer. Mol Med Rep 12: 885-894, 2015

30. Hao Y, Yang J, Yin S, Zhang H, Fan Y, Sun C, Gu J and Xi JJ: The synergistic regulation of VEGF-mediated angiogenesis through miR-190 and target genes. RNA 20: 1328-1336, 2014

31. Chen L, Li ZY, Xu SY, Zhang XJ, Zhang Y, Luo K and Li WP: Upregulation of miR-107 inhibits glioma angiogenesis and VEGF expression. Cell Mol Neurobiol 36: 113-120, 2016.

32. Li X, Zhang J, Gao L, McClellan S, Finan MA, Butler TW, Owen LB, Piazza GA and Xi Y: MiR-181 mediates cell differentiation by interrupting the Lin28 and let-7 feedback circuit. Cell Death Differ 19: 378-386, 2012.

33. Liu XD, Cai F, Liu L, Zhang Y and Yang AL: MicroRNA-210 is involved in the regulation of postmenopausal osteoporosis through promotion of VEGF expression and osteoblast differentiation. Biol Chem 396: 339-347, 2015.

34. Jiang FS, Tian SS, Lu JJ, Ding XH, Qian CD, Ding B, Ding ZS and Jin B: Cardamonin regulates miR-21 expression and suppresses angiogenesis induced by vascular endothelial growth factor. Biomed Res Int 2015: 501581, 2015.
35. Mei H, Lin ZY and Tong QS: The roles of microRNAs in neuroblastoma. World J Pediatr 10: 10-16, 2014.

36. Su YF, Zang YF, Wang YH and Ding YL: MiR-19-3p induces tumor cell apoptosis via targeting FAS in rectal cancer cells. Technol Cancer Res Treat 19: 1533033820917978, 2020.

37. Lu H, Zhang L, Lu S, Yang D, Ye J, Li M and Hu W: miR-25 expression is upregulated in pancreatic ductal adenocarcinoma and promotes cell proliferation by targeting ABI2. Exp Ther Med 19: 3384-3390, 2020.

38. Tiwari A, Mukherjee B and Dixit M: MicroRNA key to angiogenesis regulation: MiRNA biology and therapy. Curr Cancer Drug Targets 18: 266-277, 2018.

39. Yuan M, Huang LL, Chen JH, Wu J and Xu Q: The emerging treatment landscape of targeted therapy in non-small-cell lung cancer. Signal Transduct Target Ther 4: 61, 2019.

40. Zhao X, Hu GF, Shi YF and Xu W: Research progress in microRNA-based therapy for gastric cancer. Onco Targets Ther 12: 11393-11411, 2019.

41. Ryzhov S, Biktasova A, Goldstein AE, Zhang Q, Biaggioni I, Dikov MM and Feoktistov I: Role of JunB in adenosine A2B receptor-mediated vascular endothelial growth factor production. Mol Pharmacol 85: 62-73, 2014. 